\title{
Estudo de Campos Magnéticos Emitidos em Processos de Soldagem Eletrodo Revestido e TIG
}

\author{
Hélio Cardoso Martim ${ }^{1}$, Louriel Oliveira Vilarinho ${ }^{1}$ \\ ${ }^{1}$ Universidade Federal de Uberlândia - UFU, Faculdade de Engenharia Mecânica, Uberlândia, MG, Brasil
}

Recebido: 12 Abr., 2018

Aceito: 17 Ago., 2018

E-mails: helio.martim.hcm@gmail.com (HCM), vilarinho@ufu.br (LOV)
Resumo: Os processos de soldagem Eletrodo Revestido e TIG, amplamente utilizados na indústria, são executados em grande parte de forma manual e neles são empregados níveis elevados de corrente afim de garantir maior produtividade. O emprego de correntes de alta intensidade conduz a geração de altos campos magnéticos, os quais de acordo com diretrizes relacionadas a saúde e segurança do trabalhador podem acarretar em efeitos nocivos à saúde humana. Assim, foram realizadas avaliações acerca da emissão de campos magnéticos em processos de soldagem Eletrodo Revestido e TIG em função das variáveis operacionais inerentes a estes processos e o nível de exposição de soldadores através da comparação com os limites de segurança estabelecidos pelas diretrizes mais atuais do ICNIRP. Os resultados mostraram que o campo magnético é maior a medida em que aumenta a corrente de soldagem e menor quanto maior for a altura do arco. O revestimento do eletrodo e o gás de proteção também influenciam na intensidade do campo magnético por interferirem, respectivamente, na estabilidade e no formato do arco elétrico. Os resultados mostraram ainda que o campo magnético é influenciado pela permeabilidade magnética do material de base.

Palavras-chave: Eletrodo revestido; TIG; Limites de exposição.

\section{Study of Magnetic Fields Issued in Welding Processes Coated Electrode and TIG}

\begin{abstract}
Welded Coated and TIG welding processes, widely used in industry, are executed largely manually and in them are used high levels of current in order to ensure higher productivity. The use of high intensity currents leads to the generation of high magnetic fields, which according to guidelines related to the health and safety of the worker can have harmful effects on human health. Thus, evaluations were made on the emission of magnetic fields in Welded Coated and TIG welding processes in function of the operational variables inherent to these processes and the level of exposure of welders by comparison with the safety limits established by the most current ICNIRP guidelines. The results showed that the magnetic field is larger as the welding current increases and the smaller the arc height. The electrode coating and shielding gas also influence the magnetic field strength by interfering, respectively, in the stability and shape of the electric arc. The results also showed that the magnetic field is influenced by the magnetic permeability of the base material.
\end{abstract}

Key-words: Coated electrode; TIG; Exposure limits.

\section{Introdução}

Os processos de soldagem a arco são amplamente utilizados na indústria de manufatura. Como forma de elevar os níveis de produtividade e garantir a competitividade no referido cenário industrial, estão sendo empregados valores de corrente cada vez maiores [1]. Esses processos são executados de forma manual ou mecanizada com o uso de corrente alternada (AC) ou corrente contínua (DC), cujas intensidades variam de dezenas de ampères até algumas centenas de ampères. No caso dos processos Eletrodo Revestido e TIG, são comumente empregados valores de corrente que variam de 40 a $400 \mathrm{~A}$ e de 5 a 500 A, respectivamente [2].

O emprego de altos valores de corrente de soldagem acarreta na geração de campo magnético de maior intensidade [3], uma vez que o arco elétrico pode ser considerado um condutor fluídico de corrente elétrica e, portanto, sofre a interação dos campos gerados pela corrente transportada. A geração e campos magnéticos tão grandes quanto forem as intensidades de corrente empregadas no processo é explicada pela Lei de Biot-Savart, a qual expressa matematicamente através da Equação 1 , demonstra que a densidade de
Este é um artigo publicado em acesso aberto (Open Access)
sob a licença Creative Commons Attribution Non-Commerci que permite uso, distribuição e reprodução em qualquer meio, sem restrições desde que sem fins comerciais e que 0 trabalho original seja corretamente citado. 
campo magnético (B) cresce proporcionalmente com a correte de soldagem (I) e ainda com o comprimento do $\operatorname{arco}(L)$, o ângulo $(\theta)$ formado entre o vetor de campo magnético e a direção do condutor e com a permeabilidade magnética do meio $(\mu)$.

$$
B=\frac{\mu \cdot I \cdot L \cdot \operatorname{sen} \theta}{4 \cdot \pi \cdot r^{2}}
$$

A distância radial ( $r$ ) entre o condutor e o ponto onde se deseja medir ou calcular o campo magnético varia em função do formato tronco-cônico do arco. A Equação 1 revela que quanto menor for essa distância, maior será a intensidade do campo magnético, logo o campo magnético é mais intenso nas regiões próximas ao eletrodo do que nas proximidades da peça [4]. Pelo fato da intensidade do campo magnético variar diretamente com o comprimento do arco, mas inversamente com o quadrado da distância radial, arcos de menor comprimento, pelo fato de possuírem menor distância radial $(r)$, tendem a apresentar maior densidade de campo magnético do que arcos de maior comprimento.

No que diz respeito a execução de tais processos, cabe ressaltar que essas soldagens são realizadas em grande parte de forma manual, em função da sua versatilidade de aplicações. Quanto à mão de obra, é preciso levar em conta o fato de que a soldagem é um processo industrial complexo e por isso requer uma detalhada avaliação antes da fabricação, onde as atividades são regidas por normas de qualidade [5] e dessa forma, deve ser realizada por profissionais qualificados e comprometidos com a segurança ocupacional, uma vez que a maioria dos acidentes decorrem do desconhecimento dos riscos da atividade [6].

Com relação a emissão de campos magnéticos, entidades relacionadas à segurança no processo de soldagem tem se dedicado ao estudo e formulação de diretrizes que, respaldadas em evidência cientificas, limitam a exposição do trabalhador aos campos magnéticos. A International Commissionon Non-lonizing Radiation Protection (ICNIRP) é a organização que mais se destaca na referida causa. A diretiva da ICNIRP de 2002 trata dos principais efeitos nocivos à saúde humana decorrentes da exposição a campos magnéticos, dentre os quais é possível citar os efeitos no sistema nervoso central e cardiovascular, estímulo de células nervosas e musculares, aborrecimento e audição de micro-ondas. Fatos como esses justificam a elaboração de diretrizes que limitam tal exposição [7].

Para fins de proteção, a ICNIRP expressa o campo magnético como densidade de campo magnético (B), expresso em Teslas $(T)$. Segundo a diretiva mais atual com relação a exposição a campos magnéticos estáticos, 0 limite de exposição para a cabeça e tronco é de 2000 mT e de 8000 mT para os membros [8]. Quanto a exposição aos campos magnéticos variantes no tempo os limites de exposição são dados, na maior parte dos casos, em função da frequência (f) [9]. Esses limites não indicam necessariamente que, se excedidos, representam danos potenciais à saúde, mas sim valores de referência estabelecidos como medida de precaução.

Em especial para a soldagem Eletrodo Revestido e TIG, além da utilização de níveis elevados de corrente e, consequentemente, maior geração de campos magnéticos, esses processos são executados, em grande parte, manualmente, o que torna o soldador ainda mais exposto em função da redução da sua distância em relação ao arco elétrico decorrente da necessidade de estar segurando o porta-eletrodo ou a tocha de soldagem [10]. Logo, a avaliação da exposição humana a campos magnéticos em situações como essas se torna indispensável, já que que é possível encontrar evidências de que o campo magnético gerado ultrapasse os limites de exposição propostos pelo ICNIRP [11].

Na literatura, poucos são os trabalhos referentes a geração de campos magnéticos em processos de soldagem. Bowman et al. [12] estuaram a geração de campos magnéticos em frequências extremamente baixas (abaixo de $100 \mathrm{~Hz}$ ) para o processo TIG. Na soldagem TIG AC foi registrado um campo magnético na faixa de 0,0024 a 0,009 mT e 0,0004 a 0,0016 mT para TIG DC, o que sugere que os maiores valores de campo magnético estão associados aos processos realizados em corrente alternada.

Stuchly e Lecuyer [13] realizaram medições a $10 \mathrm{~cm}$ do corpo do soldador e encontraram campos magnéticos na ordem de $0,2 \mathrm{mT}, 0,3 \mathrm{mT}$ e $0,4 \mathrm{mT}$ nas proximidades da cabeça, peito e cintura dos soldadores, respectivamente. O valor do campo magnético medido próximo à mão foi bastante superior, na ordem de 1,0 mT, devido ao fato do soldador estar segurando a tocha de soldagem ou o porta-eletrodo, de acordo com o processo observado. Estes valores não foram comparados com os limites de exposição estabelecidos pela ICNIRP. Ressalta-se ainda que os 
autores não revelam precisamente em qual dos processos de soldagem, Eletrodo Revestido ou TIG, tais valores foram encontrados.

Com relação ao processo Eletrodo Revestido, Melton [2] realizou medições com um magnetômetro Hall acoplado a um sensor em pontos de medição localizado no corpo do soldador, utilizando uma fonte AC convencional e corrente de 200 A. As medições realizadas na cabeça, pescoço, tronco e cintura do soldador não ultrapassam os limites de exposição. O maior valor de campo magnético encontrado foi 0,298 mT na cintura do soldador. No entanto, ao avaliar o campo magnético gerado na mão do soldador, o valor de encontrado foi de 0,644 mT, o que ultrapassa os demais valores registrados para as demais regiões do corpo.

Levando em consideração o fato de que na maioria dos trabalhos registrados na literatura até então, a maior concentração de campo magnético se encontra na mão do soldador ou em regiões muito próximas ao arco ou a fonte responsável pela geração de tais campos, Yamaguchi-Sekino et al. [14] concentrou seu estudo em medições feitas durante processos de soldagem a arco na mão do soldador através de um magnetômetro Hall conectado a uma sonda presa ao pulso do trabalhador durante toda a jornada de trabalho. O valor do campo máximo encontrado estava na faixa de 0,35 a 3,35 mT e o valor médio por dia de trabalho foi de 0,04 a 0,12 mT.

De modo geral, nestes trabalhos não fica clara a correlação do campo magnético com a corrente de soldagem efetivamente utilizada, levando a crer que as correntes mencionadas correspondem às correntes máximas fornecidas pela fonte de energia. Os autores ainda ressaltam que as medidas foram feitas em rms (root mean square), mas os resultados também podem ser apresentados em níveis médios. Além disto, a frequência em que se analisa o campo magnético influi em seu valor. Os valores apresentados foram, na maioria dos casos, correspondentes a um harmônico de $60 \mathrm{~Hz}$, mas foi observado que alguns equipamentos produziram campo magnético mais forte em $120 \mathrm{~Hz}$ ou $180 \mathrm{~Hz}$.

Ainda é possível incluir que não são dadas informações precisas sobre o nível de corrente utilizado, material e outros parâmetros operacionais comuns aos processos de soldagem. Por esta razão este trabalho apresenta uma investigação mais precisa a respeito da emissão de campos magnéticos oriundos das soldagens Eletrodo Revestido e TIG, visto que foi realizado em condições experimentais previamente especificadas para que os resultados obtidos possam ser comparados com os limites de exposição ICNIRP de forma mais consistente.

\section{Materiais e Métodos}

Para avaliação dos campos magnéticos emitidos nos processos Eletrodo Revestido e TIG foi feito o planejamento de experimentos em função dos principais parâmetros experimentais de ambos os processos. O soldador que realizou os testes possui $1,73 \mathrm{~m}$, que corresponde a altura média de um indivíduo brasileiro do sexo masculino com idade entre 25 e 29 anos, segundo os dados do IBGE de 2008 a 2009 [15]. Durante a realização dos processos de soldagem o campo magnético foi medido nas regiões identificadas pelos números de 1 a 4 que correspondem a mão, cabeça, tronco e cintura. Tal configuração é mostrada na Figura 1 de modo semelhante à metodologia proposta nos trabalhos de [2] e [14].

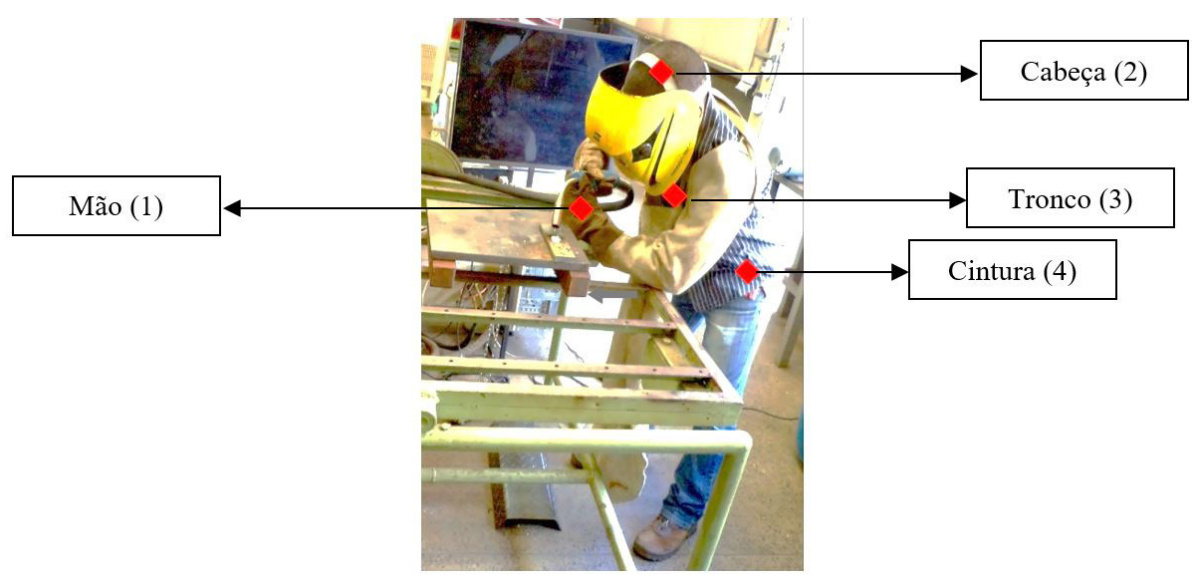

Figura 1. Configuração dos pontos de medição no soldador. 
As medições foram realizadas com o medidor de campo magnético ambiental mostrado na Figura 2a, modelo TAMB-3D, cujo fundo de escala é $5 \mathrm{mT}$ e precisão $\pm 2 \%$ da leitura. O medidor possui uma sonda magnética de 3 eixos capaz de medir a valor do campo magnético em qualquer posição [16]. Antes das medições foi efetuado o ajuste de zero colocando a blindagem de campo magnético terrestre sobre a sonda, como mostrado na Figura $2 \mathrm{~b}$.

(a)

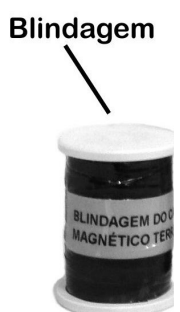

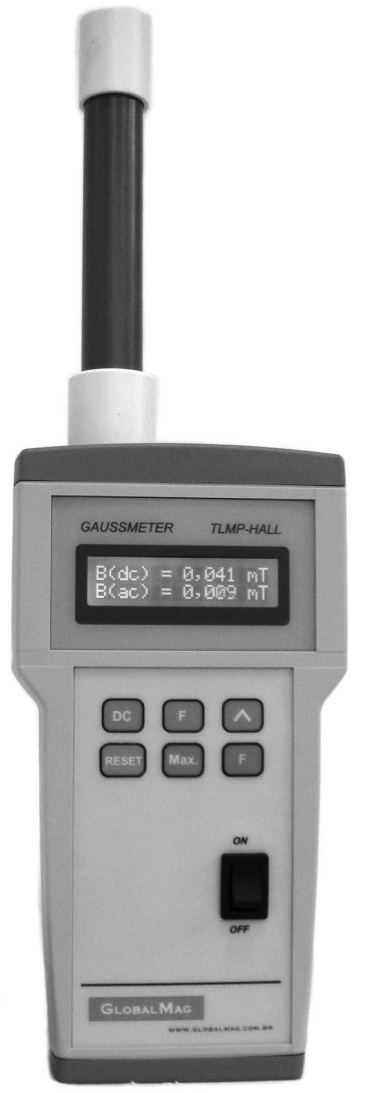

(b)

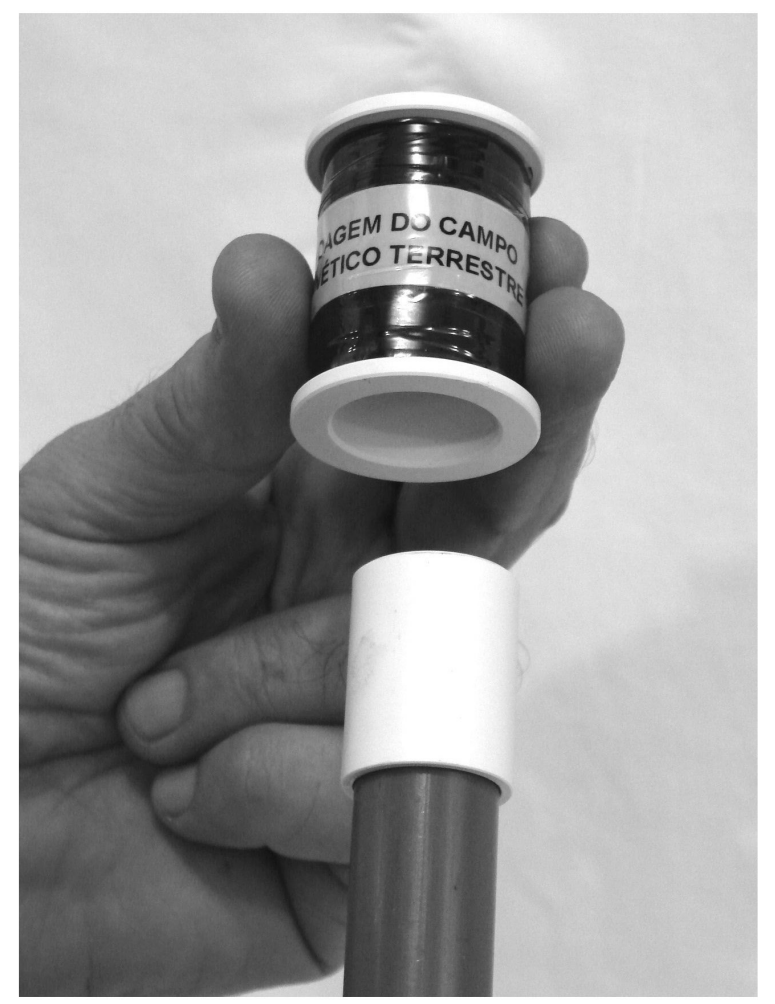

Figura 2. Medidor de campo magnético. (a) Visão geral; (b) Ajuste de zero [16].

\subsection{Medição de campo magnético para soldagem Eletrodo Revestido}

A soldagem com Eletrodo Revestido foi feita manualmente com utilização de fonte para soldagem eletrônica multiprocesso inversal da IMC, cuja faixa de corrente varia entre 0 e $300 \mathrm{~A}$ em modo corrente contínua. Quanto ao material, foram utilizadas chapas de aço carbono ABNT 1020 com dimensões de 200 x 50,2 x 12,6 mm, cuja composição e propriedades físicas estão descritas na Tabela 1 e eletrodos consumíveis celulósico E6010, rutílico E6013 e básico E7018 com diâmetro de 3,2 $\mathrm{mm}$.

Tabela 1. Composição química e propriedades mecânicas nominais do aço ABNT 1020.

\begin{tabular}{ccccc}
\hline \multicolumn{5}{c}{ Aço Carbono 1020} \\
\hline \multicolumn{5}{c}{ Composição química \% } \\
\hline $\begin{array}{c}\text { C }-0,23 \\
\text { Sensidade }-0,21\end{array}$ & Propriedades físicas \\
$\left(\mathbf{g} / \mathbf{c m}^{3}\right)$ & $\begin{array}{c}\text { Calor específico } \\
\left(\mathbf{J} / \mathbf{g}^{\circ} \mathbf{C}\right)\end{array}$ & $\begin{array}{c}\text { Condutividade } \\
\text { térmica }\left(\mathbf{W} / \mathbf{c m}^{\circ} \mathbf{C}\right)\end{array}$ & $\begin{array}{c}\text { Temperatura de } \\
\text { fusão }\left({ }^{\circ} \mathbf{C}\right)\end{array}$ & $\begin{array}{c}\text { Permeabilidade } \\
\text { magnética relativa }\end{array}$ \\
\hline 7,8 & 0,63 & 0,47 & 1520 & 100 \\
\hline
\end{tabular}


Os procedimentos experimentais foram realizados em obediência ao planejamento descrito na Tabela 2 com a utilização dos eletrodos celulósico, rutílico e básico. Os níveis alto e baixo indicam, respectivamente, 100 e 150 a para a corrente e aproximadamente 2 e $4 \mathrm{~mm}$ para a altura de arco, medidos com o auxílio de um gabarito. A faixa máxima de corrente utilizada durante esses experimentos ultrapassa a corrente máxima recomendada apenas para a soldagem com o eletrodo E6010 que é de 140 A. No entanto, foram fixados os dois níveis, baixo e alto, como forma de uniformizar os parâmetros utilizados. Em cada experimento foi medido o campo magnético nas regiões da mão, cabeça, tronco e cintura.

Tabela 2. Planejamento experimental para a soldagem Eletrodo Revestido.

\begin{tabular}{cccccccc}
\hline \multirow{2}{*}{ Ensaio } & Nível de & Altura do & \multirow{2}{*}{ Eletrodo } & \multicolumn{4}{c}{ Ponto de medição } \\
\cline { 5 - 8 } & corrente & arco & (1) & (2) & (3) & (4) \\
1 & Baixa & Baixa & Celulósico & $(1)$ & $(2)$ & $(3)$ & $(4)$ \\
2 & Baixa & Alta & Celulósico & $(1)$ & $(2)$ & $(3)$ & $(4)$ \\
3 & Alta & Baixa & Celulósico & $(1)$ & $(2)$ & $(3)$ & $(4)$ \\
4 & Alta & Alta & Celulósico & $(1)$ & $(2)$ & $(3)$ & $(4)$ \\
5 & Baixa & Baixa & Rutílico & $(1)$ & $(2)$ & $(3)$ & $(4)$ \\
6 & Baixa & Alta & Rutílico & $(1)$ & $(2)$ & $(3)$ & $(4)$ \\
7 & Alta & Baixa & Rutílico & $(1)$ & $(2)$ & $(3)$ & $(4)$ \\
8 & Alta & Alta & Rutílico & $(1)$ & $(2)$ & $(3)$ & $(4)$ \\
9 & Baixa & Baixa & Básico & $(1)$ & $(2)$ & $(3)$ & $(4)$ \\
10 & Baixa & Alta & Básico & $(1)$ & $(2)$ & $(3)$ & $(4)$ \\
11 & Alta & Baixa & Básico & $(1)$ & $(2)$ & $(3)$ & $(4)$ \\
12 & Alta & Alta & Básico & $(1)$ & $(2)$ & $(3)$ & $(4)$ \\
\hline
\end{tabular}

\subsection{Medição de campo magnético para soldagem TIG}

Para a soldagem TIG foram empregados os mesmos equipamentos do processo anterior, havendo apenas a inclusão dos cilindros de gases necessários ao processo e a substituição do porta-eletrodo pela tocha de soldagem TIGWP 18, refrigerada a água e com capacidade nominal de trabalho de $350 \mathrm{~A}$. Os testes ocorreram sem adição de material. Logo, os insumos utilizados neste processo incluem o material de base, que neste caso além do aço ABNT 1020, também foram utilizados o aço inoxidável AISI 304 e o alumínio AA5052, o gás de proteção e o eletrodo não consumível de tungstênio torinado (2\%) com 2,4 mm de diâmetro. As respectivas composições do aço inoxidável AISI 304 e do alumínio AA5052 estão dispostas nas Tabelas 3 e 4.

Tabela 3. Composição química e propriedades mecânicas nominais do aço inoxidável austenítico AISI 304.

\begin{tabular}{|c|c|c|c|c|}
\hline \multicolumn{5}{|c|}{ Aço Inoxidável Austenítico AISI 304} \\
\hline \multicolumn{5}{|c|}{ Composição química \% } \\
\hline$C-0,03$ & $M n-1,13$ & $\mathrm{Si}-0,04$ & $\mathrm{Cr}-18,06$ & $\mathrm{Ni}-8,03$ \\
\hline \multicolumn{5}{|c|}{ Propriedades físicas } \\
\hline $\begin{array}{c}\text { Densidade } \\
\left(\mathrm{g} / \mathrm{cm}^{3}\right)\end{array}$ & $\begin{array}{c}\text { Calor específico } \\
\left(\mathrm{J} / \mathrm{g}^{\circ} \mathrm{C}\right)\end{array}$ & $\begin{array}{c}\text { Condutividade } \\
\text { térmica }\left(W / \mathrm{cm}^{\circ} \mathrm{C}\right)\end{array}$ & $\begin{array}{c}\text { Temperatura de } \\
\text { fusão }\left({ }^{\circ} \mathrm{C}\right)\end{array}$ & $\begin{array}{l}\text { Permeabilidade } \\
\text { magnética relativa }\end{array}$ \\
\hline 8,0 & 0,29 & 0,033 & 1398 & 1,02 \\
\hline
\end{tabular}

Tabela 4. Composição química e propriedades mecânicas nominais do alumínio AA5052.

\begin{tabular}{|c|c|c|c|c|c|}
\hline \multicolumn{6}{|c|}{ Alumínio AA5052 } \\
\hline \multicolumn{6}{|c|}{ Composição química \% } \\
\hline $\mathrm{Al}-96,5$ & $M n-0,21$ & $\mathrm{Si}-0,25$ & $M g-2,22$ & $\mathrm{Cr}-0,32$ & $\mathrm{Fe}-0,50$ \\
\hline \multicolumn{6}{|c|}{ Propriedades físicas } \\
\hline & $\begin{array}{c}\text { Densidade } \\
\left(\mathrm{g} / \mathrm{cm}^{3}\right)\end{array}$ & $\begin{array}{c}\text { Calor específico } \\
\left(\mathrm{J} / \mathrm{g}^{\circ} \mathrm{C}\right)\end{array}$ & $\begin{array}{c}\text { Condutividade } \\
\text { térmica } \\
\left(\mathrm{W} / \mathrm{cm}^{\circ} \mathrm{C}\right)\end{array}$ & $\begin{array}{c}\text { Temperatura de } \\
\text { fusão }\left({ }^{\circ} \mathrm{C}\right)\end{array}$ & $\begin{array}{c}\text { Permeabilidade } \\
\text { magnética } \\
\text { relativa }\end{array}$ \\
\hline & 2,7 & 0,92 & 2,05 & 659 & 1,00002 \\
\hline
\end{tabular}


Nos testes de soldagem TIG foram avaliadas a corrente de soldagem em níveis baixo (100 A) e alto (150 A), a distância eletrodo-peça também em nível baixo e alto correspondendo, respectivamente a 2 e $4 \mathrm{~mm}$, gás de proteção e ponto de medição no corpo do soldador. Os gases de proteção utilizados foram o Argônio puro e uma mistura $\mathrm{Ar}+25 \% \mathrm{He}$ a uma vazão de $15 \mathrm{l} / \mathrm{min}$ ajustada com uso de um bibímetro na saída do bocal da tocha. Em cada teste realizado o campo magnético foi medido nas quatro regiões do corpo do soldador, conforme mostra o planejamento experimental descrito na Tabela 5.

Tabela 5. Planejamento experimental para a soldagem TIG.

\begin{tabular}{|c|c|c|c|c|c|c|c|c|c|}
\hline \multirow{2}{*}{ Ensaio } & \multirow{2}{*}{ Material } & \multicolumn{2}{|c|}{ Corrente } & \multirow{2}{*}{ Distância eletrodo-peça } & \multirow{2}{*}{ Gás } & \multicolumn{4}{|c|}{$\begin{array}{l}\text { Ponto de } \\
\text { medição }\end{array}$} \\
\hline & & Modo & Nível & & & (1) & (2) & (3) & (4) \\
\hline 1 & Aço C & DC & Baixo & Baixo & $\mathrm{Ar}$ & (1) & (2) & (3) & (4) \\
\hline 2 & Aço C & DC & Baixo & Alto & $\mathrm{Ar}$ & (1) & $(2)$ & (3) & (4) \\
\hline 3 & Aço C & DC & Alto & Baixo & $\mathrm{Ar}$ & (1) & (2) & (3) & (4) \\
\hline 4 & Aço C & DC & Alto & Alto & $\mathrm{Ar}$ & (1) & $(2)$ & (3) & (4) \\
\hline 5 & Aço C & DC & Baixo & Baixo & $\mathrm{Ar}+25 \% \mathrm{He}$ & (1) & (2) & (3) & (4) \\
\hline 6 & Aço C & DC & Baixo & Alto & $\mathrm{Ar}+25 \% \mathrm{He}$ & (1) & (2) & (3) & (4) \\
\hline 7 & Aço C & DC & Alto & Baixo & $\mathrm{Ar}+25 \% \mathrm{He}$ & (1) & $(2)$ & (3) & (4) \\
\hline 8 & Aço C & DC & Alto & Alto & $\mathrm{Ar}+25 \% \mathrm{He}$ & (1) & (2) & (3) & (4) \\
\hline 9 & Aço Inox & DC & Baixo & Baixo & $\mathrm{Ar}$ & (1) & (2) & (3) & (4) \\
\hline 10 & Aço Inox & DC & Baixo & Alto & $\mathrm{Ar}$ & (1) & (2) & (3) & (4) \\
\hline 11 & Aço Inox & DC & Alto & Baixo & $\mathrm{Ar}$ & (1) & $(2)$ & (3) & (4) \\
\hline 12 & Aço Inox & DC & Alto & Alto & $\mathrm{Ar}$ & (1) & (2) & (3) & (4) \\
\hline 13 & Aço Inox & DC & Baixo & Baixo & $\mathrm{Ar}+25 \% \mathrm{He}$ & (1) & (2) & (3) & (4) \\
\hline 14 & Aço Inox & DC & Baixo & Alto & $\mathrm{Ar}+25 \% \mathrm{He}$ & (1) & (2) & (3) & (4) \\
\hline 15 & Aço Inox & DC & Alto & Baixo & $\mathrm{Ar}+25 \% \mathrm{He}$ & (1) & $(2)$ & (3) & (4) \\
\hline 16 & Aço Inox & DC & Alto & Alto & $\mathrm{Ar}+25 \% \mathrm{He}$ & (1) & (2) & (3) & (4) \\
\hline 17 & Alumínio & $A C$ & Baixo & Baixo & $\mathrm{Ar}$ & (1) & (2) & (3) & (4) \\
\hline 18 & Alumínio & $A C$ & Baixo & Alto & $\mathrm{Ar}$ & (1) & (2) & (3) & (4) \\
\hline 19 & Alumínio & $A C$ & Alto & Baixo & $\mathrm{Ar}$ & (1) & (2) & (3) & (4) \\
\hline 20 & Alumínio & $A C$ & Alto & Alto & $\mathrm{Ar}$ & (1) & $(2)$ & (3) & (4) \\
\hline 21 & Alumínio & $A C$ & Baixo & Baixo & $\mathrm{Ar}+25 \% \mathrm{He}$ & (1) & $(2)$ & (3) & (4) \\
\hline 22 & Alumínio & $A C$ & Baixo & Alto & $\mathrm{Ar}+25 \% \mathrm{He}$ & (1) & (2) & (3) & (4) \\
\hline 23 & Alumínio & $A C$ & Alto & Baixo & $\mathrm{Ar}+25 \% \mathrm{He}$ & (1) & (2) & (3) & (4) \\
\hline 24 & Alumínio & $A C$ & Alto & Alto & $\mathrm{Ar}+25 \% \mathrm{He}$ & $(1)$ & $(2)$ & (3) & (4) \\
\hline
\end{tabular}

\section{Resultados e Discussão}

Os resultados obtidos para os referidos processos de soldagem são, além dos resultados de tensão e corrente adquiridos por meio de um sistema de aquisição, os valores de campos magnético avaliados estatisticamente através da análise de variância (ANOVA) feita com o nível de significância de 95\%. Isto significa que as variáveis consideradas significativas são aquelas cujo valor de $p$ é igual ou menor que $5 \%$ ( $p \leq 0,05)$. Quanto ao campo magnético, os valores oriundos dos ensaios foram posteriormente comparados com os limites de exposição estabelecidos pelas diretrizes mais atuais da ICNIRP com a finalidade de avaliar a exposição de soldadores.

\subsection{Processo eletrodo revestido}

No processo Eletrodo Revestido foram obtidos os resultados de tensão e corrente em valores médios e eficazes, bem como os valores de campo magnético medidos nas regiões do corpo, anteriormente especificadas. Esses resultados são mostrados na Tabela 6, onde se verifica uma menor corrente de soldagem no ensaio 2 , considerando uma tolerância de $\pm 5 \mathrm{~A}$, o que é aceitável em função da análise de variância (ANOVA) que, realizada com nível de significância de $95 \%(p \leq 0,05)$, mostrou-se significante $(p=0,02)$ para os valores de tensão obtidos nesses experimentos. 
Tabela 6. Medidas de campo magnético para a soldagem Eletrodo Revestido.

\begin{tabular}{|c|c|c|c|c|c|c|c|c|}
\hline \multirow{2}{*}{ Ensaio } & \multicolumn{4}{|c|}{ Campo magnético (mT) } & \multicolumn{2}{|c|}{ Corrente (A) } & \multicolumn{2}{|c|}{ Tensão (V) } \\
\hline & Mão & Cabeça & Tronco & Cintura & Média & RMS & Média & RMS \\
\hline 1 & 0,214 & 0,068 & 0,090 & 0,114 & 97 & 97 & 25,0 & 25,6 \\
\hline 2 & 0,168 & 0,065 & 0,085 & 0,107 & 91 & 91 & 36,3 & 36,6 \\
\hline 3 & 0,258 & 0,070 & 0,124 & 0,187 & 149 & 149 & 30,0 & 31,1 \\
\hline 4 & 0,241 & 0,065 & 0,097 & 0,144 & 146 & 146 & 40,4 & 40,6 \\
\hline 5 & 0,222 & 0,066 & 0,090 & 0,130 & 101 & 103 & 19,0 & 19,5 \\
\hline 6 & 0,195 & 0,064 & 0,087 & 0,124 & 100 & 102 & 18,4 & 19,0 \\
\hline 7 & 0,380 & 0,080 & 0,102 & 0,158 & 149 & 149 & 22,8 & 23,0 \\
\hline 8 & 0,300 & 0,070 & 0,102 & 0,150 & 146 & 146 & 39,2 & 39,4 \\
\hline 9 & 0,197 & 0,078 & 0,104 & 0,117 & 96 & 98 & 22,1 & 22,6 \\
\hline 10 & 0,183 & 0,073 & 0,087 & 0,109 & 98 & 100 & 22,1 & 23,0 \\
\hline 11 & 0,295 & 0,100 & 0,153 & 0,168 & 149 & 149 & 26,3 & 27,0 \\
\hline 12 & 0,268 & 0,087 & 0,143 & 0,163 & 148 & 148 & 29,8 & 30,0 \\
\hline
\end{tabular}

Assim, avaliando primeiramente o comportamento da tensão, como sendo o primeiro resultado desse grupo de experimentos, observa-se que há coerência com os dados registrados na literatura comum em função das variáveis analisadas, levando a crer que os procedimentos foram conduzidos de modo satisfatório e que as investigações acerca do campo magnético gerado nesse processo podem ser realizadas.

Quanto aos resultados de ANOVA para o campo magnético, verifica-se que todas as variáveis estudadas mostraram-se significativas quanto a sua influência na emissão de campo magnético. Os valores de campo magnético registrados nas medições especificadas (mão, cabeça, tronco e cintura) foram analisados estatisticamente em função das variáveis experimentais estudadas. A Figura 3 revela a influência da corrente de soldagem no valor de campo magnético (valores médios extraídos das medições especificadas, mediante análise estatística), o qual, em coerência com os trabalhos de Melton [2] e Ali [3], é maior quanto maior for a corrente de soldagem. Este resultado também pode ser interpretado como uma verificação experimental da lei de Biot-Savart, ao mostrar que a densidade de campo e magnético e a corrente de soldagem são grandezas diretamente proporcionais.

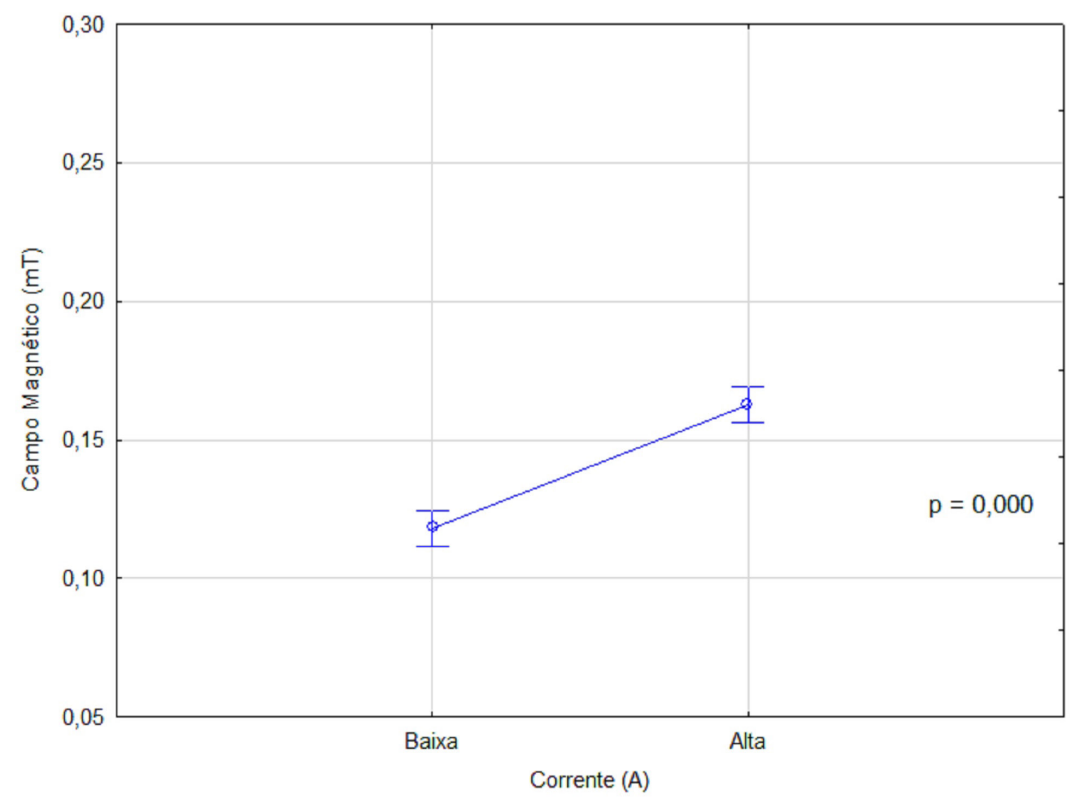

Figura 3. Influência da corrente no valor de campo magnético para o processo Eletrodo Revestido. 
A análise da lei de Biot-Savart, explica também o comportamento do campo magnético em função da altura do arco (Figura 4), uma vez que mostra que a valor do campo magnético varia diretamente com o comprimento do arco e inversamente com o quadrado da distância r, entre o condutor e ponto de medição de campo magnético. Logo, em acordo com Mair [4], arcos mais curtos, com menor distância radial e mais próximos do eletrodo, apresentam campo magnético mais intenso. Nestes arcos, a densidade de corrente também é mais elevada, o que contribui com os maiores valores de campo magnético.

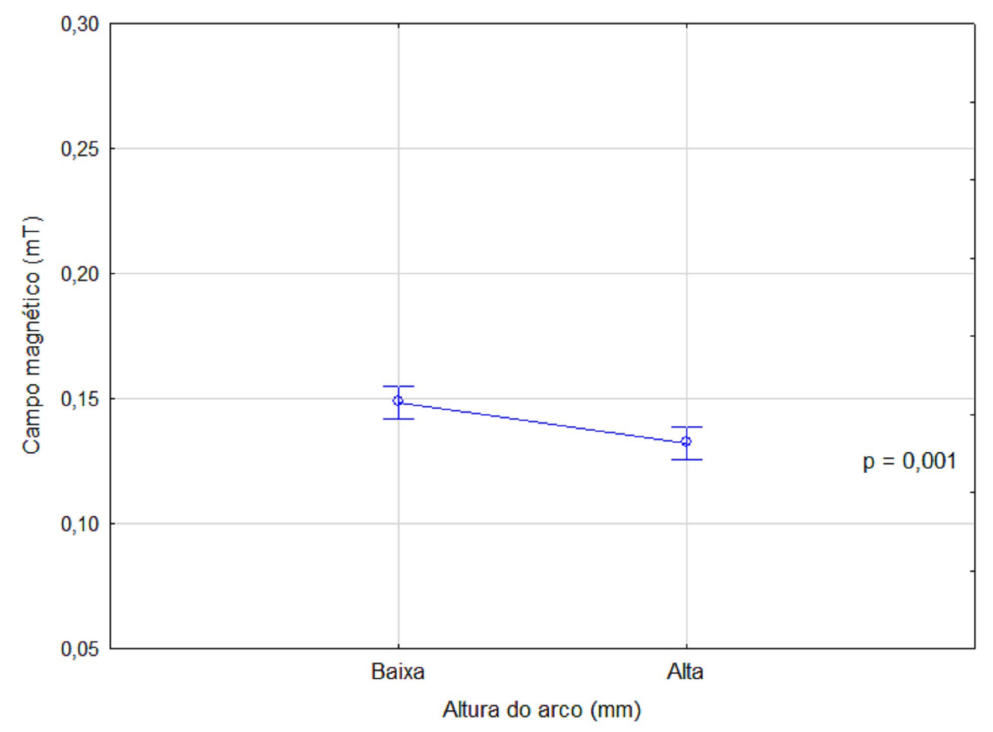

Figura 4. Influência do comprimento do arco no valor de campo magnético para o processo Eletrodo Revestido.

A Figura 5 apresenta uma comparação entre o campo magnético gerado por dois arcos de tamanhos diferentes $\mathrm{H} 1$ e H2, sendo $\mathrm{H} 1$ menor que $\mathrm{H} 2$ em um processo de soldagem realizado sob as mesmas condições. Ao aproximar o medidor do campo magnético a uma mesma distância de arcos de menor e maior comprimento é possível verificar que durante a soldagem com arcos de menor comprimento e menor distância radial um maior número de linhas de campo magnético passará pelo medidor, representando, assim, mais densidade de campo magnético.

O revestimento do eletrodo também exerce papel importante na geração dos campos magnéticos ao revelarem que este é menor na soldagem com eletrodo celulósico do que com eletrodos rutílicos e básicos, onde não se observa diferenças apreciáveis quanto a esse aspecto (Figura 6). Em virtude da ausência de dados na literatura que

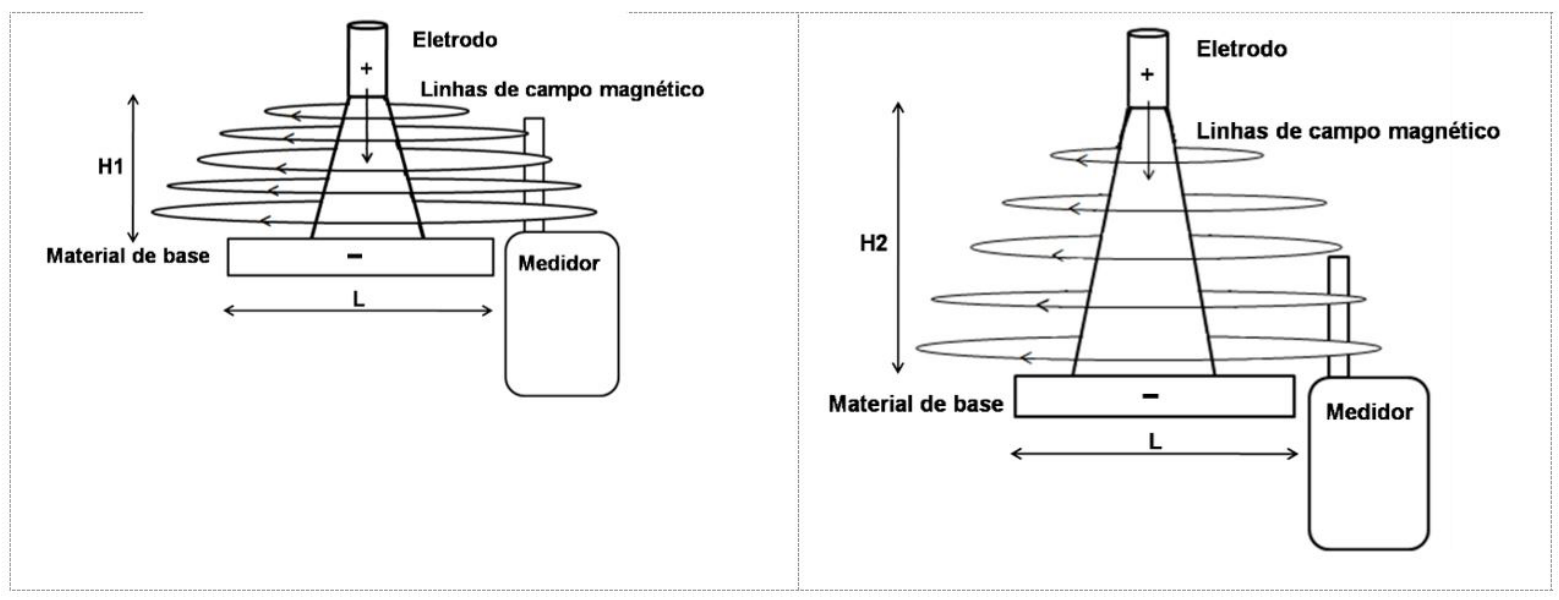

Figura 5. Representação da densidade de campo magnético em função da altura do arco de soldagem $(\mathrm{H} 1<\mathrm{H} 2)$. 


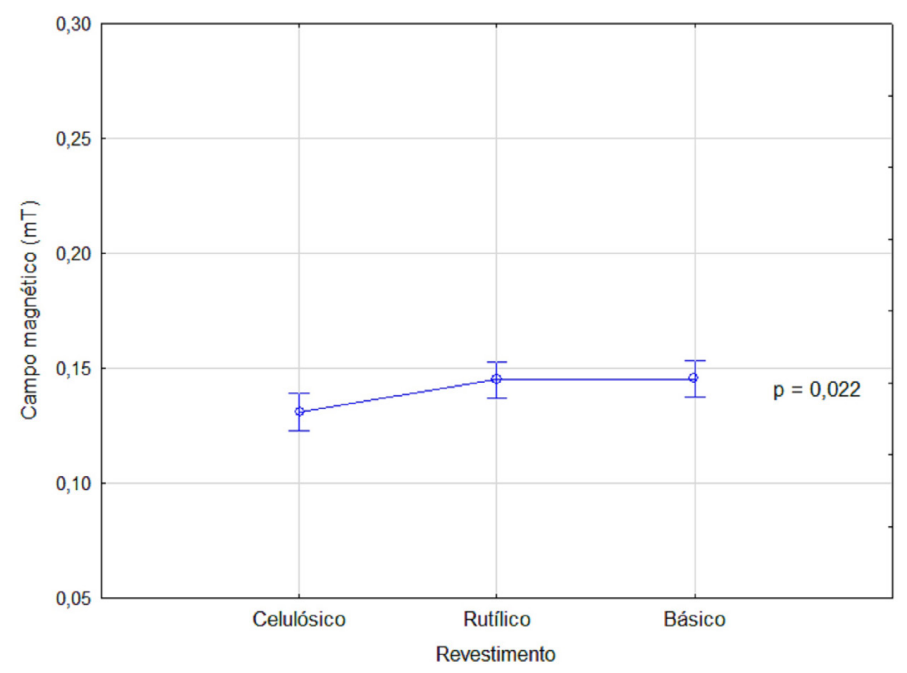

Figura 6. Influência do revestimento do eletrodo no valor de campo magnético para o processo eletrodo revestido.

justifiquem tal comportamento, acredita-se que os menores valores de campo magnético registrados na soldagem com eletrodo celulósico, ocorra em função da oscilação praticamente inexistente da corrente na soldagem com eletrodo celulósico, indicando uma transferência metálica sem curtos-circuitos. Desse modo é possível assumir que nesse caso o arco elétrico estaria maior, o que levaria a um menor campo magnético, conforme explicado na Figura 5. No entanto, para confirmar tal suposição seria necessário realizar filmagens em altas velocidades, mas que não são objetivos do presente estudo.

No que diz respeito a exposição de soldadores ao campo magnético gerado no processo com Eletrodo revestido, é possível verificar que o campo magnético é mais intenso nas regiões mais próximas ao arco, uma vez que ele aumenta inversamente com o quadrado da distância r, já especificada. Logo, como pode ser observado na Figura 7, o maior valor registrado foi na mão do soldador, pelo fato dele está segurando o porta-eletrodo e, portanto, o ponto mais próximo ao campo magnético gerado no processo. Em seguida, observa-se os valores mais elevados na região da cintura, depois do tronco e, por fim, na cabeça, região de maior distância em relação a fonte de emissão de campos magnético (arco elétrico). A heterogeneidade na distribuição de tais valores, deve-se ao fato do soldador afastar mais do arco elétrico, em função dos respingos gerados, o que causa menor valor de campo magnético no tronco e na cabeça e maior valor na cintura e na mão.

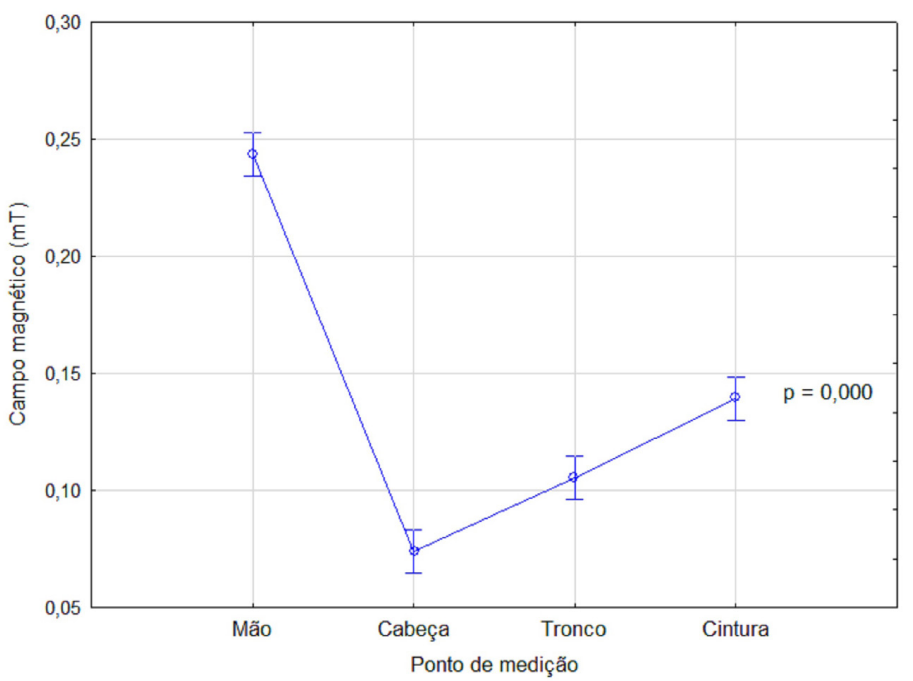

Figura 7. Influência do ponto de medição no valor do campo magnético para o processo Eletrodo Revestido. 
Os resultados obtidos para os pontos de medição mencionados, além de concordarem com os relatos de Melton [2] e Yamaguchi-Sekino et al. [14], permitem comparar com os valores limites de exposição estabelecidos pela diretiva da ICNIRP de 2009, onde conclui-se que, nas condições operatórias estabelecidas nenhum valor ultrapassa os tais limites que, segundo o referido documento, é de $2000 \mathrm{mT}$ para a cabeça e tronco e $8000 \mathrm{mT}$ para os membros.

\subsection{Processo TIG}

Bem como realizado para o processo Eletrodo Revestido, na soldagem TIG os resultados de corrente, tensão e campo magnético mostrados na Tabela 7 foram avaliados segundo análise de variância ANOVA, segundo um critério de significância de 95\% ( $p \leq 0,05)$, onde obteve-se uma significância adequada ao critério estabelecido. Os resultados para tensão, em virtude das variáveis analisadas mostram-se mais uma vez satisfatórios, uma vez que concordam com outros resultados encontrados na literatura comum para tal processo, quando executado corretamente. Sendo assim é possível prosseguir com as análises referente ao campo magnético oriundo desse processo.

Tabela 7. Medidas de campo magnético para a soldagem TIG.

\begin{tabular}{|c|c|c|c|c|c|c|c|c|}
\hline \multirow{2}{*}{ Ensaio } & \multicolumn{4}{|c|}{ Campo magnético ( $\mathrm{mT}$ ) } & \multicolumn{2}{|c|}{ Corrente (A) } & \multicolumn{2}{|c|}{ Tensão (V) } \\
\hline & Mão & Cabeça & Tronco & Cintura & Média & RMS & Média & RMS \\
\hline 1 & 0,170 & 0,063 & 0,153 & 0,065 & 100 & 101 & 10,7 & 10,7 \\
\hline 2 & 0,156 & 0,058 & 0,139 & 0,041 & 99 & 99 & 12,8 & 12,8 \\
\hline 3 & 0,319 & 0,090 & 0,302 & 0,061 & 150 & 150 & 11,8 & 11,8 \\
\hline 4 & 0,268 & 0,075 & 0,219 & 0,046 & 150 & 150 & 13,2 & 13,2 \\
\hline 5 & 0,241 & 0,063 & 0,214 & 0,058 & 99 & 100 & 11,9 & 11,9 \\
\hline 6 & 0,231 & 0,058 & 0,168 & 0,034 & 99 & 99 & 12,6 & 12,6 \\
\hline 7 & 0,370 & 0,082 & 0,275 & 0,068 & 150 & 150 & 11,0 & 11,0 \\
\hline 8 & 0,285 & 0,078 & 0,251 & 0,048 & 150 & 150 & 12,7 & 12,7 \\
\hline 9 & 0,212 & 0,051 & 0,180 & 0,039 & 100 & 100 & 11,2 & 11,2 \\
\hline 10 & 0,156 & 0,068 & 0,139 & 0,031 & 99 & 99 & 12,9 & 12,9 \\
\hline 11 & 0,268 & 0,070 & 0,226 & 0,065 & 150 & 150 & 11,1 & 11,1 \\
\hline 12 & 0,246 & 0,092 & 0,204 & 0,036 & 150 & 150 & 12,7 & 12,7 \\
\hline 13 & 0,248 & 0,043 & 0,212 & 0,036 & 99 & 99 & 11,3 & 11,5 \\
\hline 14 & 0,195 & 0,065 & 0,222 & 0,039 & 99 & 99 & 12,0 & 12,1 \\
\hline 15 & 0,307 & 0,073 & 0,273 & 0,058 & 150 & 150 & 10,8 & 10,8 \\
\hline 16 & 0,273 & 0,075 & 0,239 & 0,061 & 149 & 149 & 13,1 & 13,1 \\
\hline 17 & 0,124 & 0,051 & 0,097 & 0,031 & 86 & 104 & 6,0 & 9,9 \\
\hline 18 & 0,109 & 0,046 & 0,073 & 0,026 & 85 & 102 & 9,8 & 14 \\
\hline 19 & 0,175 & 0,070 & 0,141 & 0,046 & 128 & 153 & 6,8 & 10,5 \\
\hline 20 & 0,156 & 0,068 & 0,139 & 0,038 & 127 & 153 & 10,0 & 14,3 \\
\hline 21 & 0,129 & 0,051 & 0,117 & 0,031 & 86 & 103 & 7,0 & 11,2 \\
\hline 22 & 0,114 & 0,043 & 0,073 & 0,024 & 86 & 103 & 7,8 & 11,6 \\
\hline 23 & 0,165 & 0,070 & 0,153 & 0,046 & 128 & 154 & 7,2 & 11,3 \\
\hline 24 & 0,148 & 0,063 & 0,136 & 0,041 & 127 & 153 & 9,2 & 13,4 \\
\hline
\end{tabular}

Os valores de campo magnético mostrados em cada gráfico que segue são oriundos das medições feitas no soldador nas regiões da mão, cabeça, tronco e cintura durante a execução do processo com os parâmetros experimentais propostos. A Figura 8 mostra a influência da corrente e soldagem na intensidade de campo magnético e, bem como os resultados obtidos para o processo Eletrodo Revestido, concordam com os estudos de Melton [2], Ali [3] e Bolte e Pruppers [10] e são explicados em função da proporcionalidade existente entre a densidade de campo magnético e a corrente expressa pela Lei de Biot-Savart. Ainda, em coerência com a referida lei do eletromagnetismo e de modo semelhante aos resultados do processo anterior, a Figura 9 revela mais uma vez que arcos de menor comprimento, relativos a menor distância eletrodo- peça, conduzem a maior densidade de campo magnético em função de sua menor distância radial, $r$ [4]. 


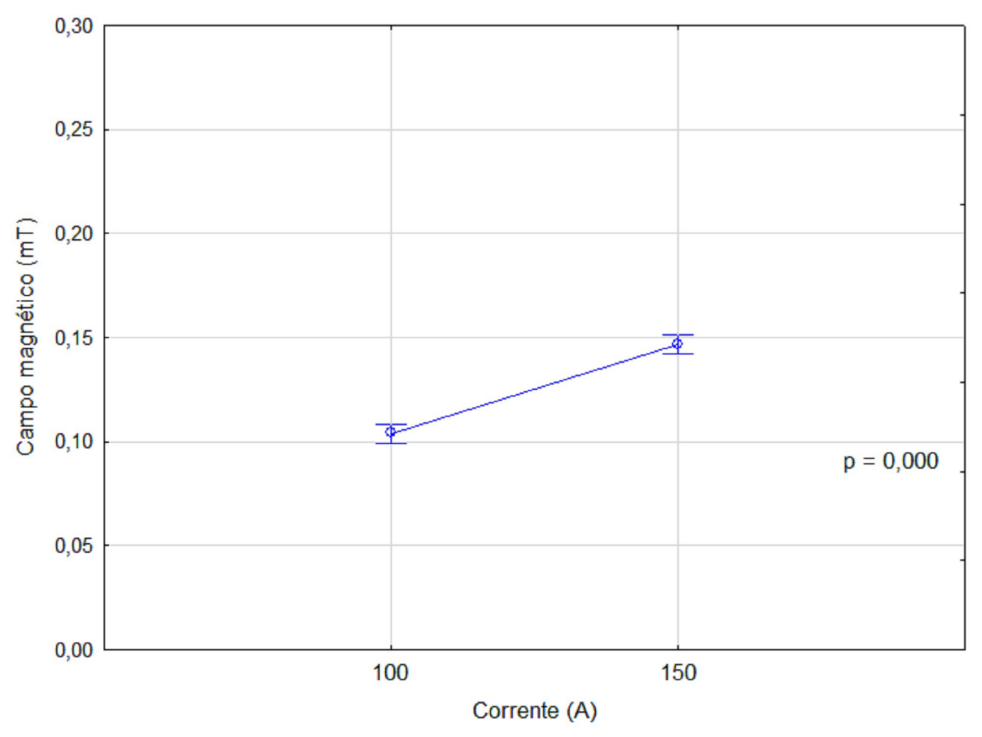

Figura 8. Influência da corrente no valor de campo magnético para o processo TIG.

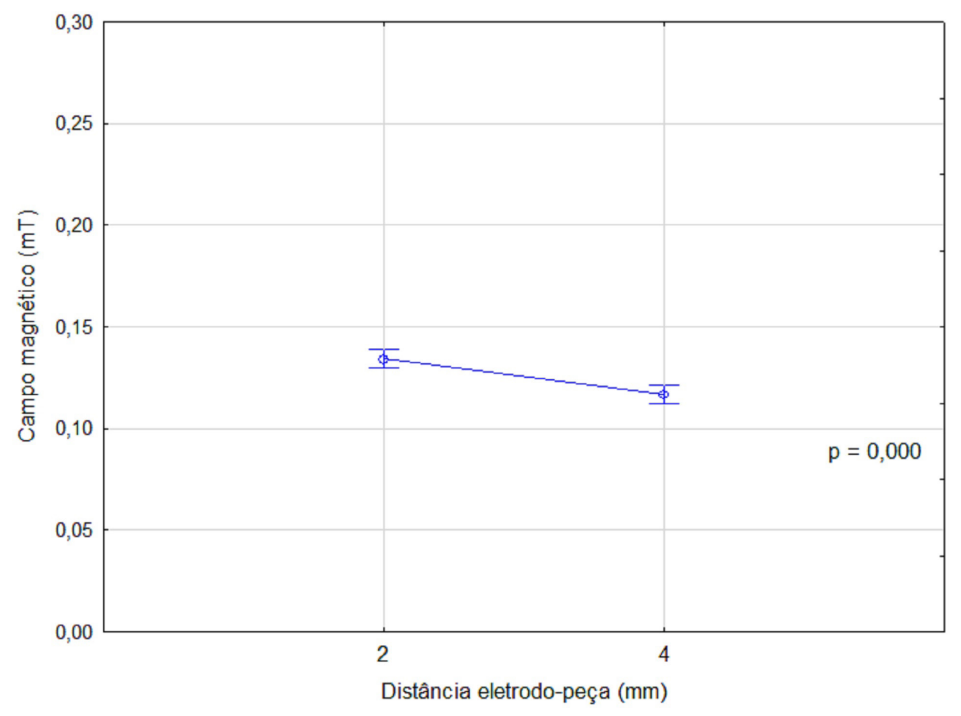

Figura 9. Influência da distância eletrodo-peça no valor de campo magnético para o processo TIG.

A proteção gasosa exerce papel importante no valor do campo magnético. Os resultados mostraram que o campo magnético é maior com a utilização da mistura gasosa $\mathrm{Ar}+25 \% \mathrm{He}$ do que com Ar puro (Figura 10). Este resultado pode ser explicado pela condutividade térmica dos gases. A condutividade térmica do He é muito superior à do Ar e esta mistura gasosa de Ar e He aumenta o aporte térmico no arco. Gases com maior condutividade térmica perdem muito calor radialmente, assumindo um formato mais estreito, enquanto gases de menor condutividade térmica possuem arcos com uma zona externa mais larga. Desse modo, sabendo que o campo magnético diminui com o quadrado da distância radial entre o condutor e ponto de medição do campo magnético gerado ao redor do arco (Lei de Biot-Savart), é possível justificar que a soldagem com proteção gasosa de Ar puro conduz a campos magnéticos de menor valor, devido ao seu formato de arco ser mais largo do que aquele formado na soldagem com $\mathrm{Ar}+25 \% \mathrm{He}$.

Quanto ao tipo de material de base, a Figura 11 revela que o maior campo magnético foi registrado para a soldagem do aço carbono 1020, seguido do aço inoxidável austenítico e, por fim, do alumínio. Acredita-se que o vapor metálico gerado durante a soldagem desses materiais influencie na permeabilidade magnética do meio $(\mu)$. 


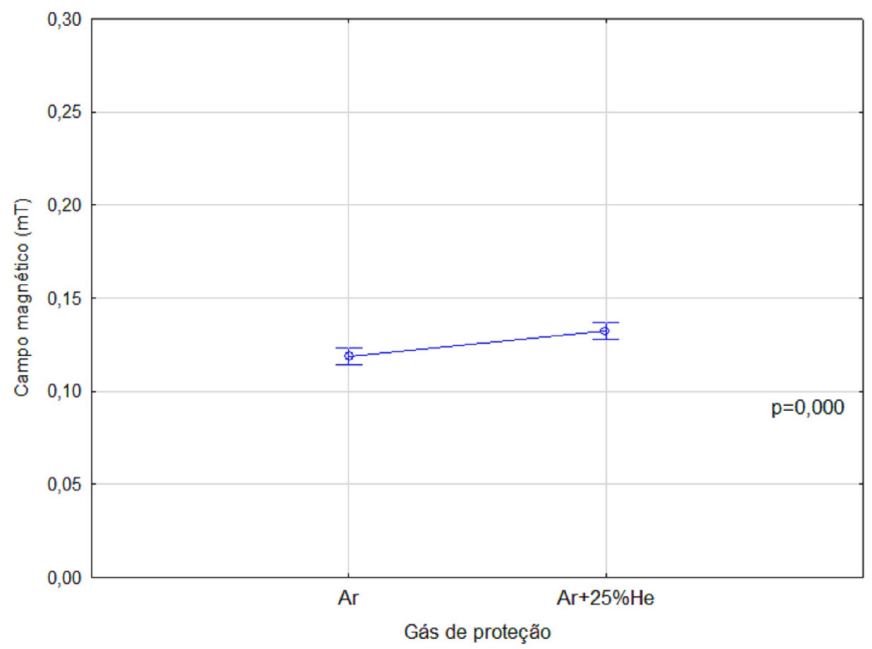

Figura 10. Influência do gás de proteção no valor de campo magnético para o processo TIG.

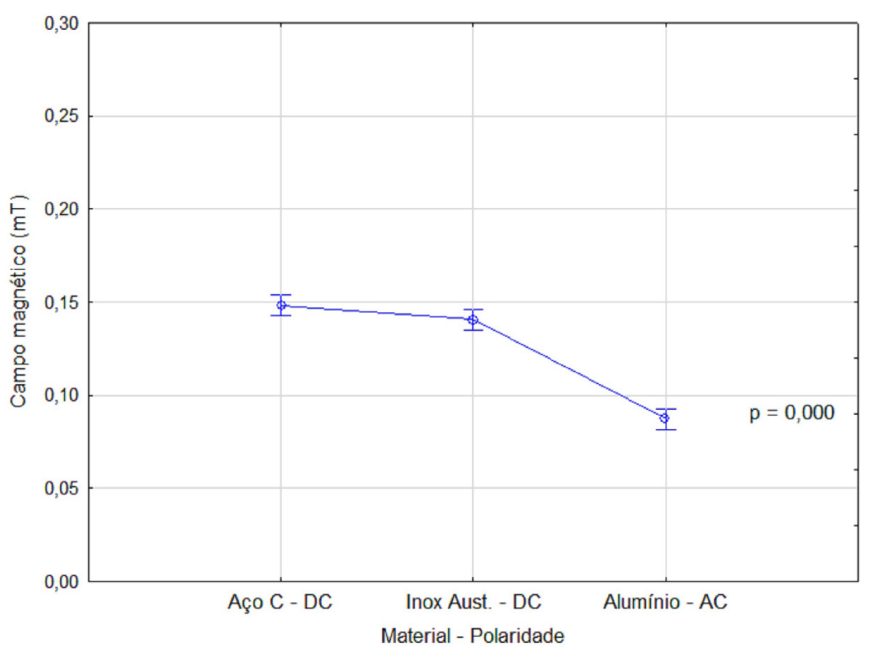

Figura 11. Influência do material de base no valor de campo magnético para o processo TIG.

Na soldagem de aços, elementos como o Ferro podem estar presentes na atmosfera e, por se tratar de um elemento ferromagnético, conduz a geração de maior campo magnético. O alumínio, no entanto, é um material paramagnético, possui permeabilidade relativa próxima ao do ar, o que leva a crer que o alumínio presente na atmosfera durante a soldagem com esse material não alterou significativamente o campo magnético gerado no processo. Ainda é indispensável ressaltar que, no caso do alumínio, em função da necessidade de limpeza catódica, a soldagem com esse material ocorreu com corrente alternada. Logo, a Figura 11 também traz informações a respeito da polaridade da corrente empregada nos processos TIG e, de acordo com os estudos de Melton [2], em soldagens com corrente alternada espera-se maiores valores de campo magnético. No entanto, como já foi exposto, no presente trabalho o modo de corrente estava associado a outras variáveis, o que torna impossível avaliar o efeito isolado da polaridade da corrente no referido processo de soldagem.

Assim como no processo Eletrodo Revestido, no processo TIG também foi verificado uma distribuição heterogênea dos valores de campo magnético nas diferentes regiões do corpo do soldador. Em concordância com os trabalhos de Bowman et al. [12] e Stuchly e Lecuyer [13], na mão do soldador foi registrado o maior valor campo magnético, em virtude na necessidade de segurar a tocha de soldagem durante o processo manual e, portanto, ser 
o ponto de maior proximidade ao arco elétrico. Nos demais pontos de medição os resultados foram diferentes dos encontrados no processo anterior. Na soldagem TIG após a mão, os maiores valores de campo magnético foram encontrados no tronco, seguido da cabeça e por fim na cintura (Figura 12). Esta diferença pode ser atribuída ao fato do soldador mudar de posição de um processo para outro. Percebe-se que na soldagem TIG o soldador inclinou-se em direção a tocha de soldagem, aproximando o tronco e a cabeça e consequentemente afastando a cintura.

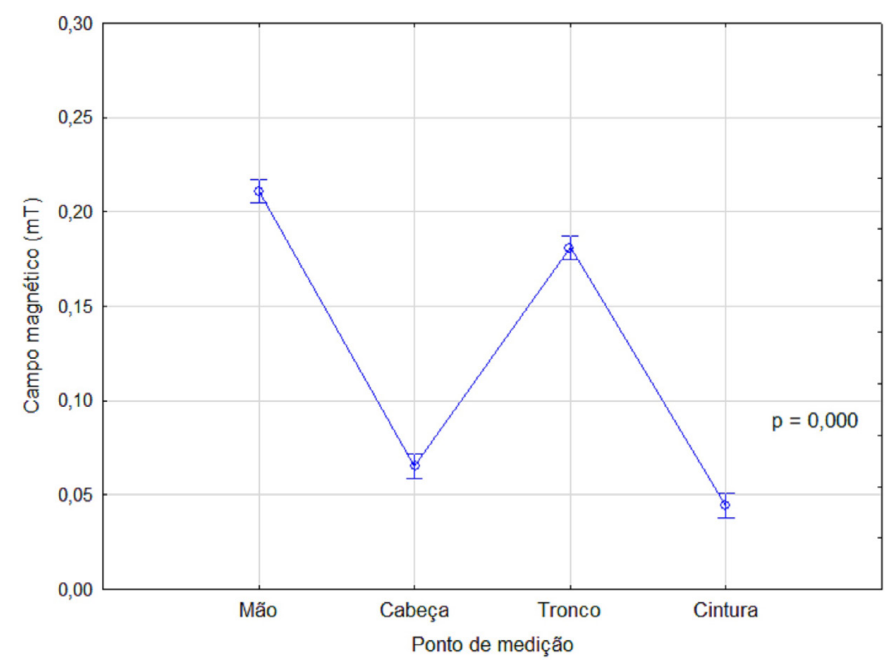

Figura 12. Influência do ponto de medição no valor de campo magnético para o processo TIG.

A comparação dos valores de campo magnético medidos com os limites de exposição regulamentados pela ICNIRP deve ser feita em dois momentos. O primeiro refere-se aqueles valores obtidos no modo de corrente contínua que devem ser comparados com os limites de exposição registrados na ICNIRP de 2009 (2000 mT para a cabeça e tronco e $8000 \mathrm{mT}$ para membros) por se tratar de campos estáticos. O segundo diz respeito ao campo magnético mensurado nas soldagens com corrente alternada, devendo ser comparada com limites de exposição contidos da diretiva ICNIRP de 2010, referente a campos magnéticos variantes no tempo. De acordo com esta última diretiva, para os processos TIG AC feitos neste trabalho, cuja nível de frequência foi de $40 \mathrm{~Hz}$, o valor de referência é de $1 \mathrm{mT}$. A análise geral dos resultados revela que os valores obtidos variam de 0,031 mT na cintura a 0,370 mT na mão do soldador, mas em nenhum processo de soldagem TIG, seja ele AC ou DC, os valores de referência foram ultrapassados.

\section{Conclusões}

Diante do que foi exposto, é possível verificar que a metodologia utilizada na realização dos experimentos é adequada, uma vez que foi capaz de revelar resultados coerentes com aqueles registrados na literatura ou justificáveis a partir de teorias do eletromagnetismo ou por estudos de outros autores. A partir de tais resultados, portanto, percebe-se que a corrente de soldagem é, de fato, o fator predominante quanto a influência na geração de campos magnéticos. Aqueles processos em que foram empregados corrente de maior valor apresentaram também campo magnético mais intenso.

Quanto ao que se observa no processo Eletrodo Revestido, conclui-se que o aumento na altura do arco representa geração de campos magnéticos de menor valor em função do formado cônico do arco de soldagem e que soldagens com eletrodos de revestimento celulósico produzem campos magnéticos menores que aqueles gerados em soldagem com eletrodos rutílicos e básicos.

No que se refere ao processo TIG, soldagens realizadas com proteção gasosa $\mathrm{Ar}+25 \% \mathrm{He}$ geram campo magnético mais intenso do que aquelas realizadas com Ar puro, em função do formato do arco assumido com este tipo de gás de proteção. A mudança do material de base também implica em geração de diferentes valores de campo magnético. Soldagens feitas com aço carbono 1020 apresentam campo magnético maior que soldagens 
feitas com aço inoxidável austenítico em corrente contínua. Em ambos os processos o valor de campo magnético é superior ao registrado em soldagens com alumínio no modo corrente alternada.

Em ambos os processos, percebe-se que quanto maior a altura do arco menor é o valor de campo magnético, o qual também apresenta valores não uniformes nas diferentes regiões do corpo do soldador em função da sua distância ao arco elétrico. Assim, tanto no processo eletrodo revestido, como no processo TIG, registram-se os maiores valores de campo magnético na mão do soldador, pelo fato dele está segurando o porta-eletrodo ou a tocha de soldagem. Quanto as demais regiões, do corpo, no processo eletrodo revestido o segundo maior valor de campo magnético encontra-se na cintura, seguido do tronco e cabeça. No processo TIG os maiores valores de campo magnético depois da mão, estão no tronco, cabeça e cintura, em ordem decrescente. Apesar das diferenças existentes entre os valores de campo magnético, explicadas em função da mudança de posição do soldador ao executar os processos, nenhum dos valores registrados ultrapassam os limites de exposição estabelecidos pelas diretivas do ICNIRP, dentro das condições operatórias descritas no presente estudo.

\section{Agradecimentos}

Ao CnPq pelo apoio financeiro e ao Laprosolda da Universidade Federal de Uberlândia.

\section{Referências}

[1] Shimizu H, Itoh K, Masaie N, Kurokawa T, Ushio M. Feedability of wires during metal active gas welding. Science and Technology of Welding and Joining. 2006;11(1):81-93. http://dx.doi. org/10.1179/174329306X77876.

[2] Melton GB. Measurement and analysis of magnetic fields from welding processes. Norwich: Health \&Safety Executive; 2005. 51 p. TWI Research Report 338.

[3] Ali KJ. Measurement of magnetic fields emitted from welding machines. Diyala Journal of Engineering Sciences. 2012;5(2):114128.

[4] Mair P. Assessment of EMF (electromagnetic fields) and biological effects in arc welding applications. In: International Institute of Welding. Commission 12th, Intermediate Meeting, Fronius International; 2005; France. France: IIW; 2005. p. 1-10.

[5] International Organization for Standardization - ISO. ISO 3834:2005. Quality requirements for fusion welding of metallic materials. Geneva: ISO; 2005. 7 p.

[6] Silva MLF, Souza JS, Siqueira AMA. Análise qualitativa dos riscos químicos inerentes à saúde no ambiente laboral que abrange o processo de soldagem com eletrodos revestidos. In: Anais do $34^{\circ}$ Encontro Nacional de Engenharia de Produção; 2014; Curitiba. Curitiba: ABEPRO; 2014. p. 1-7.

[7] International Commission on Non-Ionizing Radiation Protection - ICNIRP. General approach to protection against non-ionizing radiation protection. Health Physics. 2002;82(4):540-548. http://dx.doi.org/10.1097/00004032-200204000-00017. PMid:11906144.

[8] International Commission on Non-Ionizing Radiation Protection - ICNIRP. Guidelines on limits of exposure to static magnetic fields. Health Physics. 2009;96(4):504-514. http://dx.doi. org/10.1097/01.HP.0000343164.27920.4a. PMid:19276710.
[9] International Commission on Non-Ionizing Radiation Protection - ICNIRP. Guidelines for limiting exposure to time - varying electric, magnetic and electromagnetic fields (up to $300 \mathrm{GHz}$ ). Health Physics. 1998;74(4):494-522. PMid:9525427.

[10] Bolte JFB, Pruppers MJM. Electromagnetic fields in the working environment. Utrecht: RIVM; 2006. Report of ministry of social affairs and employment.

[11] Grassi F, Spadacini G, Pignari SA. Human exposure in arc-welding processes: current versus previous ICNIRP basic restrictions. In: Proceedings of the IEEE International Symposium on Electromagnetic Compatibility (EMC); 2012 Aug 6-10; Pittsburgh, Pennsylvania. USA: IEEE; 2012. http://dx.doi.org/10.1109/ ISEMC.2012.6350920.

[12] Bowman JD, Garabrant DH, Sobel E, Peters JM. Exposure to extremely low frequency (ELF) electromagnetic fields in occupations with elevated leukemia rates. Appliedindustrial Industrial Hygiene. 1988;3(6):189-194.

[13] Stuchly MA, Lecuyer DW. Exposure to electromagnetic fields in arc welding. Health Physics. 1989;56(3):297-302. http://dx.doi. org/10.1097/00004032-198903000-00003. PMid:2917858.

[14] Yamaguchi-Sekino S, Ojima J, Sekino M, Hojo M, Saito H, Okuno T. Measuring exposed magnetic fields of welders in working time. Industrial Health. 2011;49(3):274-279. http://dx.doi. org/10.2486/indhealth.MS1269. PMid:21670555.

[15] Instituto Brasileiro de Geografia e Estatística - IBGE. Pesquisas de orçamentos familiares 2008-2009 [página de internet]. Rio de Janeiro: IBGE; 2015 [acesso em 10 out. 2015]. Disponível em: http://www.ibge.gov.br

[16] Globalmag. Medidor de campo magnético ambiental: TAMB3D. Cotia: GLOBALMAG; 2015. Manual de operação. 